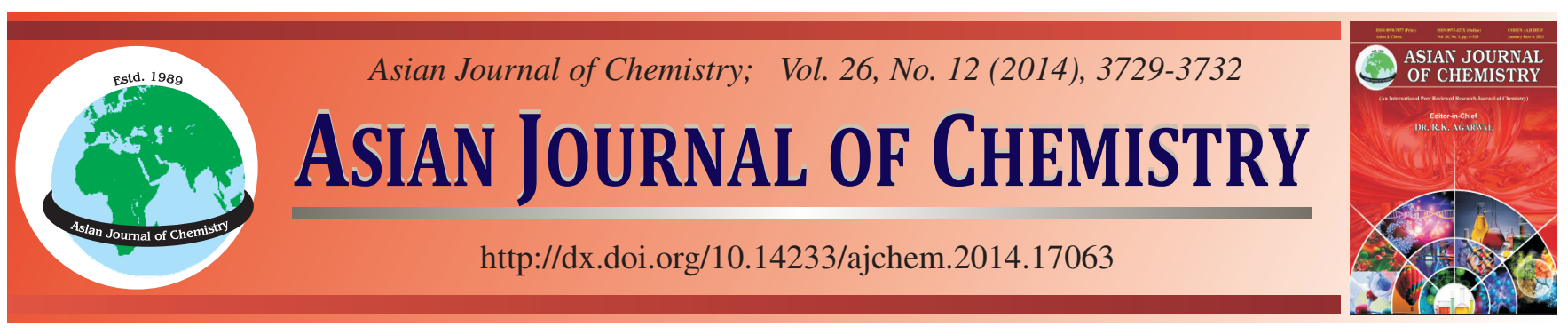

\title{
Extraction and Purification of C-Phycocyanin from Spirulina platensis Using Aqueous Two Phase Extraction and its Applications $\dagger$
}

\author{
Senthilkumar Rathnasamy*, J. Jennet Debora and B.N. Vedha Hari
}

School of Chemical and Biotechnology, SASTRA University, Thanjavur-61 340, India

*Corresponding author: E-mail: rsenthilkumar@biotech.sastra.edu

Spirulina platensis is a highly potential Cyanobacteria (blue green algae) that acts as immuno-modulator. Present study deals with the extraction of phycocyanin from this potent cyanobacteria employing non organic method. Organic denaturation and protein instability encountered in the conventional organic solvent extraction method can be overcome by biological extraction method. Phycocyanin, a phycobiliprotein present in the Spirulina extracted using aqueous two phase extraction and it was compared with isoelectric precipitation. The resulting fractions were spectrometrically assayed for phycocyanin content. The fraction with high phycocyanin content was identified and purified using anionic exchange chromatography. The resulting purified fraction was evaluated for antibacterial activity and antioxidant activity.

Keywords: Spirulina platensis, Phycocyanin, Aqueous two phase extraction, Anionic purification antimicrobial activity.

ᄂ - - - - - - - - - - - - - - - - - - - - - - - - - - - - -

\section{INTRODUCTION}

In the recent years, Spirulina a cyanobacterium is attracting attention due to its nutritional values besides being highly economical ${ }^{1}$. In addition to proteins they contain essential vitamins, minerals, fatty acids and chlorophyll, certain coloured compounds like carotenoids and chlorophyl1 ${ }^{2,3}$. Spirulina are filamentous cyanobacterium that can be monocultured and harvested easily ${ }^{4}$. It is widely used in food industry, cosmetics and pharmaceuticals as colourants and as a substitute of synthetic dyes. Spirulina is reported to possess antioxidant, antiinflammatory and anticancer activity.

Phycobiliproteins are group of proteins present in Spirulina as they are similar to the bile pigments bilirubin and biliverdin. Phycobiliproteins form a light harvesting antenna complexes called the phycobilisomes which are attached to the thylakoid membrane ${ }^{5}$. Phycobilisomes are responsible for light absorption $^{6}$. Phycobiliproteins are highly stable, water soluble proteins and have high therapeutic value. Phycocyanin (C-PC), phycoerythrin (PE) and allophycocyanin (APC) are the three types on phycobiliproteins ${ }^{7}$. Phycocyanin is abundantly present phycobiliprotein in Spirulina. Allophycocyanin also constitute to the total phycobiliprotein content of Spirulina and present in the ratio of 1:10 phycobiliproteins are the major constituents of the marine pico phytoplankton and are responsible for the total carbon biomass ${ }^{8}$. Phycocyanin role in molecular biology is becoming highly significant as it has replaced ethidium bromide which is carcinogenic and highly expensive, but phycocyanins are safe and economical ${ }^{9}$. The chromophore prosthetic group in phycocyanin account for the fluorescent properties of these proteins.

Aqueous two phase extraction (ATPE) is one of the best economical methods used in the present study for protein extraction. Aqueous two phase extraction is carried with the help of polymer-polymer system or a polymer-salt system. Polyethylene glycol (PEG) is the most commonly used polymer. Earlier gelatin soluble starch and gelatin agar were used. Potassium phosphate, ammonium sulfate and sodium citrate salts are frequently used in aqueous two phase extraction ${ }^{10}$. Addition of polyethylene glycol and salt at a particular composition, results in the formation of two phases due to the incompatibility that exist between the polymer and the salt ${ }^{11-14}$. Even the polymer utilized in the forward extraction can be recycled by back extraction procedure. Aqueous two phase extraction is a less expensive method to extract protein with high purity. In ATPE method protein gets separated in the polymer rich phase (top phase) in the forward reaction. The top phase with the polymer and protein is separated by further analysis. The protein molecules are the top phase can be separated from the polymer to the bottom phase by adding sodium chloride to 
the separated top phase sample ${ }^{15}$. Forward extraction retains only phycocyanin at the top phase while the other contaminants including allophycocyanin get separated at the bottom phase.

\section{EXPERIMENTAL}

Crude preparation: The Spirulina platensis was cultured in Zarrouk's medium and at the end of the cultivation period the biomass was obtained which was subjected to freeze drying. The frozen sample was finely ground into a fine powder and sieved.

Phycocyanin extraction: The powdered sample was mixed in phosphate buffer with $\mathrm{pH} 7$ which contained potassium dihydrogen phosphate and dipotassium hydrogen phosphate. 0.01 sodium azide was added as a preservative. The mixture was incubated over night at $4{ }^{\circ} \mathrm{C}$ to allow the lysis of the cells. At the end of the incubation period the mixture was centrifuged at $10000 \mathrm{rpm}$ for $10 \mathrm{~min}$. The blue supernatant was obtained and the above procedure was repeated until no more phycobiliprotein is present in the supernatant. The supernatant was checked for phycocyanin concentration according to the equation proposed by Bennet and Bogorad ${ }^{16}$.

$$
\text { Phycocyanin }(\mathrm{mg} / \mathrm{mL})=\frac{\left(\mathrm{A}_{620 \mathrm{~nm}}-\left(0.474 \times \mathrm{A}_{652 \mathrm{~nm}}\right)\right.}{5.34}
$$

Aqueous two phase extraction method (ACPE): The aqueous two phase system was performed with $\mathrm{PEG}$ of various molecular weight such as $(2000,4000,6000,8000 \mathrm{w} / \mathrm{v})$. Polyethylene glycol and ammonium sulfate were used based on phase diagram. Initially PEG was dissolved well in the crude sample by gently vortexing in all the five different concentrations in different test tubes. To all these test tubes initially a salt concentration of $20 \%$ was added to each tube and mixed thoroughly and left for phase separation. Once two phases were identified, the samples were transferred to a centrifuge tube and were centrifuged for better separation. Phycocyanin concentration $^{16}$ of the two phases was determined. The reference for each system was prepared by just neglecting the addition of crude Spirulina extract, the same protocol was followed for all the different molecular weights of PEG and salt concentrations.

Study of partition coefficient: The partition coefficient (K) was studied for all the systems above using the following formula

$$
\mathrm{K}_{\text {part }}=\frac{\mathrm{PC}_{\text {top }}}{\mathrm{PC}_{\text {bot }}}
$$

The system with highest $\mathrm{K}$ value is chosen as the optimum system for phycocyanin separation and that system was chosen for back extraction.

Backward extraction: The top phase of the system with highest $\mathrm{K}$ value was taken and to this equal volume of $1.2 \mathrm{M}$ sodium chloride solution was added. Sample was kept for $0.5 \mathrm{~h}$ and observed for the phycocyanin separation to the bottom phase from the top of PEG phase. Then the sample in the bottom phase was also separated for further studies.

Isoelectric precipitation method: The extracted crude sample was precipitated using $2 \mathrm{~N}$ glacial acetic acid. The extracted sample was taken to which prepared glacial acetic acid was added drop wise and the $\mathrm{pH}$ was checked after every drop. The acid was added until the proteins gets precipitated and the $\mathrm{pH}$ was measured at the point which corresponds to the isoelectric point of phycocyanin. The precipitated sample was centrifuged and the pellet was dissolved in a phosphate buffer for the estimation of phycocyanin concentration.

Purification by anion exchange chromatography: The phycocyanin content obtained by both the bioseparation method were compared. The method from which highly concentrated phycocyanin was obtained is used for further purification. As the protein is negatively charged it was purified with anion exchange chromatography which contains a positively charged solid support. The ligand used was Q sepharose where Q is Quaternary amine. $20 \mathrm{Mm}$ Tris $\mathrm{HCl}$ of $\mathrm{pH} 7.4$ was the binding buffer used and the column was eluted with the help of $0.5 \mathrm{M}$ $\mathrm{NaCl}$.

Native PAGE: The fraction that is obtained from the anion exchange chromatography is subjected to native PAGE for finding the molecular weight of the protein.

Antibacterial activity: The antibacterial activity of the purified phycocyanin sample was evaluated against seven different bacterial culture using agar well diffusion method. The organisms used are Zymomonas mobilis, Escherichia coli, Staphylococcus aureus, Bacillus cereus, Klebsiella pneumoniae, Salmonella typhi and Pseudomonas aeruginosa. Chloramphenicol was used as control.

Antioxidant activity: Antioxidant potential of phycocyanin was determined through reducing power assay. The purified sample of varying concentration was taken in different test tubes. To this $2.5 \mathrm{~mL}$ of phosphate buffer and $2.5 \mathrm{~mL}$ of $5 \%$ potassium ferricyanide was added and incubated in a water bath at $50{ }^{\circ} \mathrm{C}$ for $20 \mathrm{~min}$. The above mixture was centrifuged at $10000 \mathrm{rpm}$ for $2 \mathrm{~min}$. From the supernatant $2.5 \mathrm{~mL}$ was separated to which $2.5 \mathrm{~mL}$ of distilled water and $0.5 \mathrm{~mL}$ of 15 $\%$ ferric chloride was added. The samples were observed for colour change and absorbance was measured at $680 \mathrm{~nm}$.

\section{RESULTS AND DISCUSSION}

Bioextraction: The extraction step has a key role in the extraction of proteins in their natural state. The crude was extracted using different $\mathrm{pH}$, of which phycocyanin content was found to be high at a $\mathrm{pH}$ range of 6.8-7.2. The concentration of phycocyanin was determined spectrometrically according to the equation proposed by Bennet and Bogorad ${ }^{16}$. Phycocyanin has a maximum absorption at $620 \mathrm{~nm}$. The absorbance of the extract at $280 \mathrm{~nm}$ was found to be higher than at $620 \mathrm{~nm}$ which indicates the presence of other proteins besides phycocyanin.

Aqueous two phase extraction: The protein concentration and the volume in the top and bottom phase were calculated. ATPE enables the differential partitioning of phycocyanin to the top phase and allophycocyanin to the bottom phase. The fractionation of phycocyanin to the polymer phase is due to its high affinity to PEG, which also resulted in a high purity of phycocyanin. Absorbance of the top phase was high at 620 $\mathrm{nm}$ due to phycocyanin fractionation and the maximum absorbance of the bottom phase was observed at $652 \mathrm{~nm}$ which is attributed to allophycocyanin. As aqueous two phase extrac- 
tion is mainly used for concentrating the protein sample, a system with high concentration of phycocyanin is taken into consideration. The partition coefficient for all the system was calculated. Fig. 1a-b gives the variation of partition coefficient with varying PEG molecular weight and concentration.
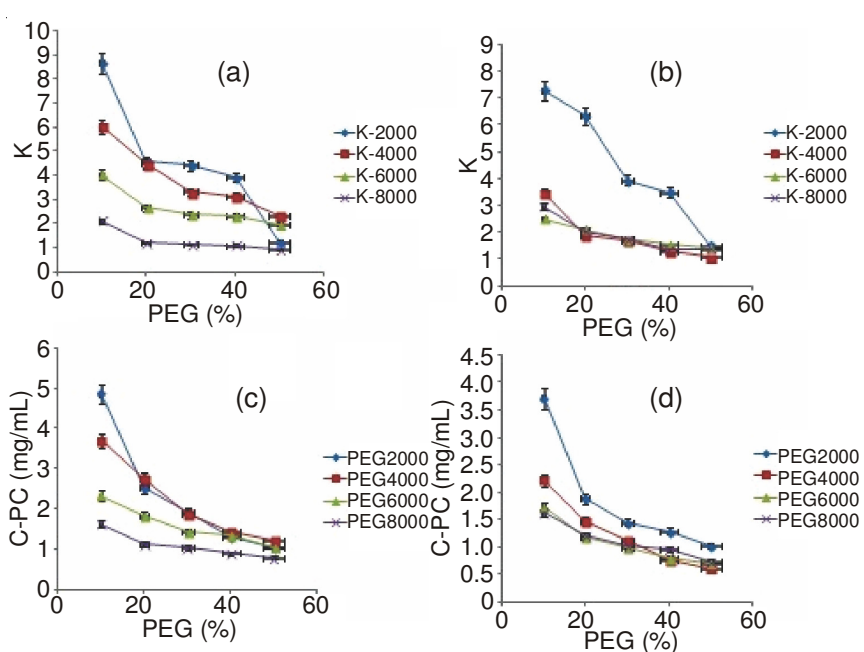

Fig. 1. a,b: Effect of PEG \% molecular weight concentration on partition coefficient; c,d: Effect of PEG \% molecular weight concentration on C-phycocyanin (C-PC) $\mathrm{mg} / \mathrm{mL}$

The system with molecular weight of PEG 2000 and PEG concentration of $10 \%$ and ammonium sulfate concentration of $20 \%$ had the highest partition coefficient. As the salt concentration increases the partition coefficient of the system was found to decrease which indicates that higher salt concentration reduces the separation of the system.

Effect of phase composition: Changes in the phase composition have a direct influence on the physical properties such as interfacial tension, viscosity and density because of which variation in solute partitioning takes place. Changes in free volume are reported to be the reason behind the variation in solute partitioning, the increase in the interfacial tension with the phase composition is attributed to the difference in the phase composition of the top and bottom. The composition of polymer and salt were selected in such a way that the system would lie above the bimodal curve to ensure better separation. As the concentration of PEG increases the partition coefficient decreases. Recovery of phycocyanin to the top phase is because of the volume exclusion effect.

Effect of polymer molecular weight: Variation of PEG molecular weight has a wide influence on the partition coefficient. As the molecular weight of PEG increases there is a decrease in the partition coefficient which is because of the decrease in the free volume in the top phase. Effect of polymer molecular mass in the partitioning behaviour depends on the protein molecular mass. The contribution of PEG molecular mass is less significant in proteins of molecular mass less than $50 \mathrm{KDa}$ and is highly significant in proteins of molecular weight more than $50 \mathrm{KDa}$. In the present study phycocyanin, which was reported to have molecular weight greater than $50 \mathrm{KDa}$ was found to have a higher partition coefficient in PEG2000. Fig. 1c-d clearly depicts that highly concentrated phycocyanin was obtained in PEG 2000 with $10 \%$ PEG concentration.
Effect of pH: Variation in the $\mathrm{pH}$ alters the protein surface charge. This is because of the changes that occur in the ionizable groups of the protein molecules. Change in $\mathrm{pH}$ alters the partitioning of the protein and the partition coefficient varies exponentially. The phycocyanin extraction was carried out at $\mathrm{pH} 7$ at which the protein will be negatively charged and prefer the top phase for separation.

Effect of volume ratio: Partitioning of the protein to the top phase takes place when the phase volume ratio increases due to which free volume decreases in the bottom phase, as the phase volume ratio increases the partition coefficient increases which indicates efficient protein transfer to the top phase. In aqueous two phase extraction, proteins with low partition coefficient can be separated to the top phase at high phase volume ratio.

Backward extraction: The above selected system was processed for back extraction. Backward extraction is mainly employed to recycle the polymer that is used in forward extraction. This feature of aqueous two phase extraction was a breakthrough in bioseparation processes which makes it a highly economical process. The addition of $\mathrm{NaCl}$ of $1.2 \mathrm{M}$ resulted in the back extraction of the phycocyanin. $\mathrm{NaCl}$ of varying molarity was used for the same system. Initially it was started with $0.5 \mathrm{M} \mathrm{NaCl}$ solution. Complete separation of PEG and phycocyanin was achieved at $1.2 \mathrm{M} \mathrm{NaCl}$. This is because concentrations lower than $1.2 \mathrm{M}$ is not sufficient to separate the protein molecules from PEG.

Isoelectric precipitation: The isoelectric point is the point at which the protein molecules do not contain any net charge, which results in reduced solubility of the protein molecules in the medium. Adding glacial acetic acid to the crude phycocyanin extract at this point resulted in precipitation of protein at $\mathrm{pH} 4.6$ which corresponds to the isoelectric point of phycocyanin. The precipitated sample was centrifuged and the pellet obtained was dissolved in phosphate buffer for protein estimation.The protein concentration was found to be high in the pellet. The low protein content in the supernatant is due to the fact that it's only the phycocyanin which got separated but not the allophycocyanin which still remains in the buffer and its isoelectric point is different from that of phycocyanin.

The phycocyanin extraction using the two bioseparation methods namely aqueous two phase extraction and isoelectric precipitation is compared based on the phycocyanin concentration obtained in the two methods. Aqueous two phase extraction resulted in an efficient extraction of phycocyanin both in terms of concentration as well as purity. Aqueous two phase extraction coupled with backward extraction was successful in obtaining phycocyanin in an economical way. Although, application of isoelectric precipitation for phycocyanin extraction is encouraging, a highly concentrated extract was obtained in case of ATPE only. The phycocyanin concentration was calculated based on the equation proposed by Bennet and Bogorad $^{16}$.

A single peak was obtained in an anion exchange column which clearly indicates that the presence of contaminants is very less as shown in Fig. 2a. The choice of buffer $\mathrm{pH}$ is very important because in case of anion exchange column decreasing the $\mathrm{pH}$ of the buffer makes the protein less negatively charged and therefore it cannot bind properly to the column. In the 


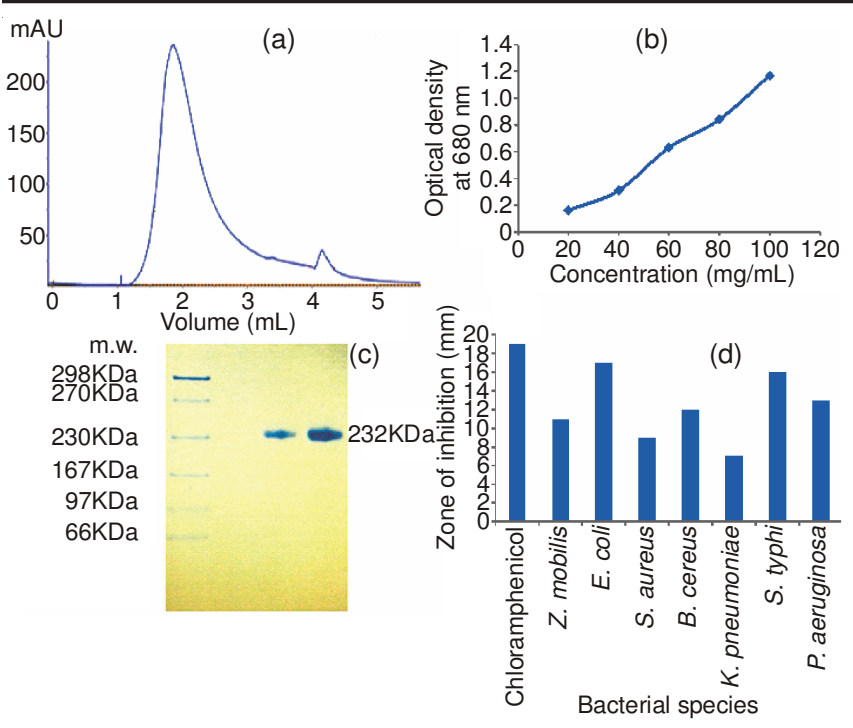

Fig. 2. a. C-PC ion exchange chromatography; b. Antioxidant assay, c. SDS page analysis, d. Antibacterial study

same way in cation exchange column, increasing the $\mathrm{pH}$ of the buffer makes the protein molecules less positively charged and so it cannot bind properly to the column. Therefore, for phycocyanin which is negatively charged, binding buffer of $\mathrm{pH} 7.4$ was used .The samples were eluted from the column and the eluted sample was used for further study.

Native PAGE: The native-PAGE carried out for the phycocyanin sample, helped to find the molecular weight of phycocyanin. Lane 1 consists of standard molecular marker, lane 2 and lane 3 consists of the purified phycocyanin sample. A single peak was obtained for lane 2 and lane 3 which indicate that there are no other protein molecules that are present in the sample other than phycocyanin. To find the molecular weight of phycocyanin it was compared with the marker. From Fig. 2c, the molecular weight of phycocyanin was found to be $232 \mathrm{KDa}$.

Of the seven microbial species screened, phycocyanin was found to have maximum antibacterial activity against E.coli followed by Salmonella typhi. Phycocyanin was found to have an excellent anti-bacterial activity. The control used was chloramphenicol which is a bacteriostatic antimicrobial agent. From Fig. 2d, it was found that phycocyanin had minimum antibacterial activity against Klebsiella pneumonia. As the concentration of the sample increases the absorbance increases which indicates the reducing potential (antioxidant activity) of phycocyanin. The colour change is because of the conversion of $\mathrm{Fe}^{3+}-\mathrm{Fe}^{2+}$. Therefore the free radical gets converted to more stable products and the termination of free radical initiated chain reaction, that takes place is presented in Fig. $2 b$.

\section{Conclusion}

In the present study the extraction of phycocyanin is carried out with two bioseparation methods. Study proved aqueous two phase extraction is the best method for extraction. The reason behind choosing aqueous two phase for the extraction of protein is, because it is highly economical and moreover the PEG that is been used for the extraction can be recycled for further process as it could be recovered in the backward extraction. The sample was efficiently purified in anion exchange chromatography. The purified phycocyanin extract was found to possess excellent antibacterial and antioxidant properties.

\section{REFERENCES}

1. F.S. Antelo, A. Anschau, J.A.V. Costa and S.J. Kalil, J. Braz. Chem. Soc., 21, 921 (2010).

2. P. Prabuthas, S. Majumdar, P.P. Srivastav and H.N. Mishra, J. Stored Prod. Postharvest Res., 2, 93 (2011).

3. M. Rito-Palomares, J. Chromatogr. B, 807, 3 (2004).

4. G. Patil, S. Chethana, M.C. Madhusudhan and K.S.M.S. Raghavarao, Bioresour. Technol., 99, 7393 (2008).

5. V. Kumar, A.K. Bhatnagar and J.N. Srivastava, J. Med. Plants Res., 5, 7043 (2011).

6. C. Romay, R. Gonzalez, N. Ledon, D. Remirez and V. Rimbau, Curr. Protein Pept. Sci., 4, 207 (2003).

7. S.K. Mishra, A. Shrivastav and S. Mishra, Process Biochem., 43, 339 (2008).

8. Y. Zhu, X.B. Chen, K.B. Wang, Y.X. Li, K.Z. Bai, T.Y. Kuang and H.B. Ji, Appl. Microbiol. Biotechnol., 74, 244 (2007).

9. J.-F. Niu, G.-C. Wang and C.-K. Tseng, Protein Expr. Purif., 49, 23 (2006).

10. R.B. Roman, J.M. Alvarez-Pez, F.G.A. Fernandez and E.M. Grima, J. Biotechnol., 93, 73 (2002).

11. M. Rito-Palomares, J. Chromatogr. B, 807, 3 (2004).

12. G. Patil, S. Chethana, M.C. Madhusudhan and K.S.M.S. Raghavarao, Bioresour. Technol., 99, 7393 (2008).

13. A.K. Vinay Kumar, J. Med. Plants Res., 5, 7043 (2011).

14. K.G. Sabarinathan, G. Ganesan, Eur. Rev. Med. Pharmacol. Sci., 12, 79 (2008).

15. Y. Zhu, X.B. Chen, K.B. Wang, Y.X. Li, K.Z. Bai, T.Y. Kuang and H.B. Ji, Appl. Microbiol. Biotechnol., 74, 244 (2007).

16. A. Bennett and L. Bogorad, J. Cell Biol., 58, 419 (1973). 